\title{
Band head spin for triaxial super-deformed bands in ${ }^{165,167} \mathrm{Lu}$
}

\author{
Poonam Jain ${ }^{1, \star}$ and Samit K. Mandal ${ }^{2}$ \\ ${ }^{1}$ Amity Institute of Nuclear Science and Technology (AINST), Amity University, U.P., INDIA \\ ${ }^{2}$ Department of Physics and Astrophysics, University of Delhi, New Delhi-110007, INDIA
}

\begin{abstract}
We use VMI model for the prediction of band head spin of Triaxial SuperDeformed (TSD) rotational bands. The calculated and observed transition energies are agreed well when an accurate band head spin $\left(I_{0}\right)$ is predicted. The results are in good agreement with the experimentally known values of spin and transition energies. In the present paper, we have reported the band head spin of TSD bands for Lu isotope. This method brings comprehensive interpretation for spin assignment of TSD bands which could help in designing future experiments for these bands. Thus, we have reported the band head spin value of 5 TSD rotational band of $\mathrm{Lu}$ isotope.
\end{abstract}

\section{Introduction}

The study of Triaxial Super-Deformed nuclei has become an exciting field in the nuclear structure. The nuclei which has large triaxial deformation parameter $\gamma$ and large quadrupole deformation $\epsilon_{2}$ known as Triaxial Super-Deformed $(T S D)$ nuclei [1,2].

Until now, enough data for axial superdeformed nuclei are accumulated but the data for TSD nuclei are quite few like ${ }^{163} \mathrm{Lu},{ }^{164} \mathrm{Lu},{ }^{165} \mathrm{Lu},{ }^{167} \mathrm{Lu},{ }^{171} \mathrm{Ta},{ }^{86} \mathrm{Zr}$ etc. were identified experimentally. The prediction of TSD nuclei in $A \sim 160$ region has been reported in Ref. [2]. However, Bohr and Mottelson predicted the existence of the nuclear wobbling motion in rapidly rotating triaxially deformed systems [3]. The TSD nuclei has the configuration with $\epsilon_{2} \sim 0.4$ and $|\gamma| \sim 20^{0}$ has been well known in a Lu isotope since long [4]. For the first time, an excited TSD band was reported in ${ }^{163} \mathrm{Lu}[5]$.

Various empirical or semi-empirical models have been proposed for the spin assignments in SD bands. Unfortunately, very few experiments could perfectly assign spins of SD bands. The theoretical modeling is only technique to calculate the spin values. Several efforts for assigning spins of SD states have been proposed in $A \sim 190$ mass region.

Further some of the methods were proposed to assign band-head spins and transition energies as a function of spin or rotational frequency $\omega$. However, the spin assignments in these studies were not in good agreement with the experimental values. Uma et al. [6] have proposed a modified Variable Moment of Inertia (VMI) model to predict the band head spin of the superdeformed bands. In this paper a modified Variable Moment of Inertia $(V M I)$ model is used to assign band-head spin of TSD bands directly from experimentally observed transition energy ratio which requires less computation time $[7,8]$. The extension of the VMI model to an asymmetric $\left(\gamma_{0}\right)$ rotator can only be done by an

^e-mail: poonam.jn1@gmail.com 
additional assumption. The method given is reasonable, since it reduces to the usual VMI model for $(\gamma=0)$. But the results are not changed appreciably with respect to the procedure given [9]. In this model, transition energy ratio $(\mathrm{R})$ is a function of two parameters that is restoring force constant $(C)$ and moment of inertia $\left(\mathcal{J}_{0}\right)$. Both values are computed by a best-fitting method incorporating experimentally known transition energies with spin values and verified by r.m.s. deviation. It help to assign band-head spin correctly.

Thus, we organize the paper as : A brief description of theoretical and experimental efforts of superdeformed and triaxial superdeformed rotational band in nuclei is presented in the paper. In Sec 2, we briefly describe the formalism for assigning band-head spin of TSD rotational bands using modified VMI model. The results of these models are presented and compared in Sec 3 for the TSD bands. Finally Sec 4, contains a conclusion and brief remarks.

\section{A brief description of the modified VMI model}

Here we use modified VMI model to explain the relation of rotational energy with angular momentum $(I)$ for each level. It is basically the sum of the potential energy term $\left(\mathcal{J}_{I}-\mathcal{J}_{0}\right)$ and rotational energy term $\left[\left(\frac{\hbar^{2}}{2 \mathcal{J}_{I}}\right) \cdot I(I+1)\right]$. However, modified VMI model is a two-parameter formula which characterizes each nucleus by band-head moment of inertia $\left(\mathcal{J}_{0}\right)$ and restoring force constant $(C)$. The parameters depend on the difference in moment of inertia $\left(\mathcal{J}_{I}\right)$ from that of the ground-state moment of inertia $\left(\mathcal{J}_{0}\right)$. The energy level $\left(I_{0}=0\right)$ of rotational band is represented as:

$$
E_{I}(\mathcal{J})=\frac{1}{2} C\left(\mathcal{J}_{I}-\mathcal{J}_{0}\right)^{2}+\frac{1}{2}\left[\frac{I(I+1)}{\mathcal{J}_{I}}\right] .
$$

Here we assume $\hbar=1$. While the band head energy level $\left(I_{0} \neq 0\right)$ of rotational band is given as:

$$
E_{I}=E_{0}+\frac{1}{2 \mathcal{J}_{I}}\left[I(I+1)-I_{0}\left(I_{0}+1\right)\right]+\frac{1}{2} C\left(\mathcal{J}_{I}-\mathcal{J}_{0}\right)^{2}
$$

where $E_{0}$ is the band-head energy of the rotational band. The variable moment of inertia $\mathcal{J}_{I}$ is determined by the equilibrium condition.

$$
\frac{\partial E\left(\mathcal{J}_{I}\right)}{\partial \mathcal{J}_{I}}=0
$$

This is further equivalent to the cubic equation,

$$
\mathcal{J}_{I}^{3}-\mathcal{J}_{I}^{2} \mathcal{J}_{0}-\left(\left[I(I+1)-I_{0}\left(I_{0}+1\right)\right] / 2 C\right)=0 .
$$

The cubic equation has one real root for any finite positive value of $\mathcal{J}_{0}$ and $C$. Finally the transition energy for TSD bands is defined as [6]:

$$
E_{\gamma(I \rightarrow I-2)}=\frac{1}{2 \mathcal{J}_{0}}[I(I+1)-(I-2)(I-1)]+\frac{1}{8 C \mathcal{J}_{0}^{4}}\left([I(I+1)]^{2}-[(I-2)(I-1)]^{2}\right) .
$$

In this equation, the parameters $\left(\mathcal{J}_{0}\right)$ and $(C)$ are determined by fitting the experimentally known transition energies using the Best-Fit Method (BFM). The band head spin is determined in terms of the observed transition energy ratio. The transition energy ratio $(R)$ can be expressed as:

$$
R=\frac{E_{\gamma}(I+2 \longrightarrow I)}{E_{\gamma}(I \longrightarrow I-2)} .
$$

Hence the band head spin $I_{0}$ is determined from R. 
The root mean square deviation (r.m.s.) of calculated transition energies for different $I_{0}$ values are calculated and shown in figures. The r.m.s. value is least for band-head spin value of a band. If $I_{0}$ value shifts away by \pm 1 from the accurate value, a rapid shift in r.m.s. deviation $(\chi)$ can be seen. The rms deviation is taken as [6],

$$
\chi=\left[\frac{1}{n} \Sigma\left|\frac{E_{\gamma}^{c a l}\left(I_{i}\right)-E_{\gamma}^{\exp }\left(I_{i}\right)}{E_{\gamma}^{\exp }\left(I_{i}\right)}\right|^{2}\right]^{\frac{1}{2}} .
$$

Where $n$ is the total number of fitted transitions involved. Lowest spin $I_{o}$ is predicted from the least value of r.m.s., as $I_{o}$ is known all the other spin values of the TSD band levels can be determined. It is clear that the fitting procedure is quite simple and straightforward [6]. Therefore, the experimentally observed band head spin for TSD bands are well justified with the $I_{0}$ predicted from VMI equations.

\begin{tabular}{|c|c|c|c|}
\hline ISD Band & \begin{tabular}{|l|} 
Stiffness \\
Constant \\
Cx10? \\
$($ (kev)
\end{tabular} & $\begin{array}{l}\text { Band } \\
\text { Head } \\
\text { Moment of inertia } \\
\text { (J) (keV } 1 \text { ) }\end{array}$ & $\begin{array}{c}\text { Presentassigned } \\
\text { (VMI) } \\
\text { lo }\end{array}$ \\
\hline 165 Lu (b1) & 4.69 & 0.0718 & 12.5 \\
\hline 100 Lu (b3) & 234 & 0.065 & 20.5 \\
\hline 100 Lu (b1) & 1.63 & 0.0598 & 12.5 \\
\hline${ }_{16}$ Lu (b2) & 5.62 & 0.0687 & 17.5 \\
\hline $16 /$ Lu (b3) & 2.32 & 0.0651 & 13.5 \\
\hline
\end{tabular}

Table 1. The two parameters stiffness constant $(C)$ and band head moment of inertia $\left(\mathcal{J}_{0}\right)$ used in the fitting along with predicted band head spin $\left(I_{0}\right)$ using modified VMI model are given.

It can be observed that a correct spin value of the lowest TSD level, $I_{o}$, is assigned when the calculated transition energy is almost same with the observed transition energy. However, if $I_{o}$ is shifted from the correct value, even by \pm 1 the r.m.s. deviation will no longer be least i.e. there is a increase in the r.m.s. deviation with the shift in band head spin. The band head spins of all the five TSD bands have been successfully predicted by this approach. Therefore, the V-shaped $\chi-I_{0}$ plot gives a reasonable assignment of $I_{0}$.

\section{Results}

In this section, we predicted the band head spin of Lu isotopes using modified VMI model. The fitting is suceedingly done for ${ }^{165} \mathrm{Lu}(b 1, b 3)$ and ${ }^{167} \mathrm{Lu}(b 1, b 2, b 3)$ isotopes, where $b 1, b 2$ and $b 3$ refer to band 1 , band 2 and band 3 respectively. We present our results in Table 1 and 2 . The experimental data are taken from Ref. [10]. 


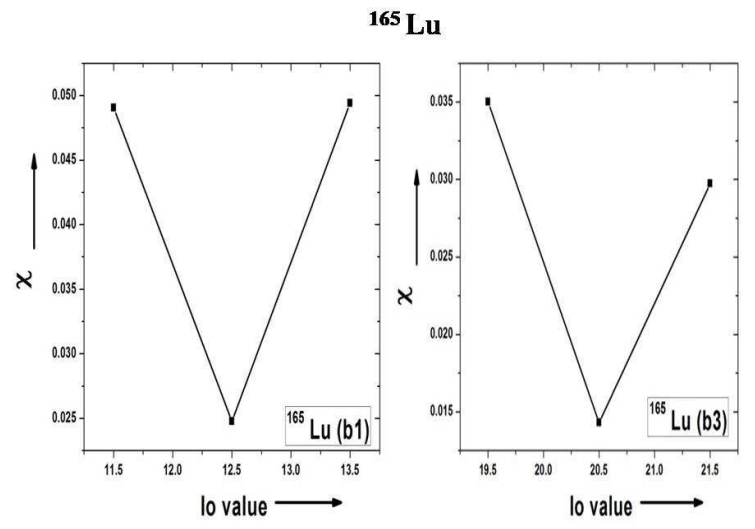

Figure 1. The r.m.s. deviation plots showing the minimum value for band head spin for ${ }^{165} \mathrm{Lu}$

The two parameters, band head moment of inertia $\mathcal{J}_{0}$ and restoring force constant $(C)$ are determined by fitting experimentally known transition energies using Best Fit Method $(B F M)$ and the cubic equation for Lu isotopes are given in Table 1.

Using the parameters adopted in Table 1, we calculated the transition energies and with the help of calculated transition energies we are able to predict the band head spin of selected cases. The obtained gamma energies are in good agreement with the experimentally known values of energies.

\begin{tabular}{|c|c|c|c|c|c|}
\hline TSD Band & 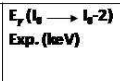 & 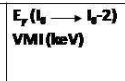 & \begin{tabular}{|l} 
Band Head \\
(L) \\
Exp.
\end{tabular} & $\begin{array}{l}\text { Assigned (b) } \\
\text { from VMI }\end{array}$ & $\begin{array}{c}\text { rms. } \\
\text { deviation } \\
\text { (x) }\end{array}$ \\
\hline $105 \mathrm{Lu}$ (b1) & 385 & 392 & 12.5 & 12.5 & 0.0248 \\
\hline $165 \mathrm{Lu}$ (b3) & 661 & 651.4 & 20.5 & 20.5 & 0.0143 \\
\hline 107 Lu (b1) & 471 & 455 & 12.5 & 12.5 & 0.063 \\
\hline $10 \mathrm{Lu}$ (b2) & 547 & 547.6 & 17.5 & 17.5 & 0.0073 \\
\hline 16' Lu (b3) & 457 & 452.7 & 13.5 & 13.5 & 0.0238 \\
\hline
\end{tabular}

Table 2. Experimental transition energies, calculated transition energies using modified VMI model along with experimental and predicted band head spin are given for 5 TSD bands for $\mathrm{Lu}$

We present our results in Table 2 that gives the experimental transition energy $E_{\gamma}$, calculated transition energy, experimental band head spin and predicted band head spin using VMI equation. It 
is also noticed that r.m.s. deviation is minimum for these bands which is shown in Figure 1 and 2 for ${ }^{165} \mathrm{Lu}$ and ${ }^{167} \mathrm{Lu}$ respectively. The predicted band head spin is compared with experimentally known spin values which provide a significant validity of this approach for TSD bands also. The results are encouraging. Overall, the obtained spin values are well justified by the r.m.s. deviation.

${ }^{167} \mathbf{L u}$
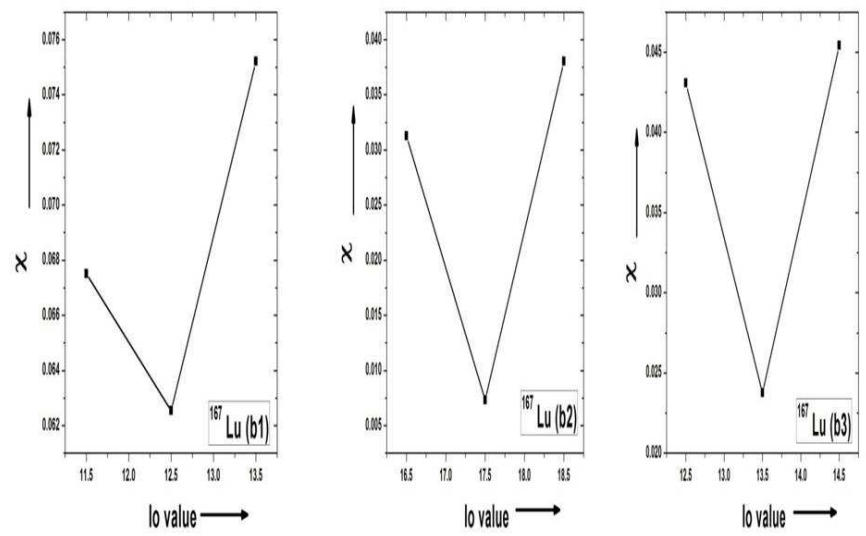

Figure 2. The r.m.s. deviation plots showing the minimum value for band head spin for ${ }^{167} \mathrm{Lu}$

\section{Conclusion}

We have predicted the band head spin of 5 TSD bands in Lu isotope. Our good results for the spins and transition energies governs that the fitting parameters $(C)$ and $\left(\mathcal{J}_{0}\right)$ obtained here for these TSD bands are acceptable and the simple model employed here is appropriate to use for TSD bands. More profound analysis of TSD bands will be published in future.

\section{References}

[1] H. Schnack-Petersen et al., Nucl. Phys. A 594, 175 (1995).

[2] D. G. Sarantites et al., Phys. Rev. C 57, (1998) R1.

[3] A. Bohr and B. R. Mottelson, Nuclear Structure Benjamin, Vol. II, (1975).

[4] W. Schmitz, et al., Phys. Lett. B 303, 230 (1993).

[5] S. W. Odegard, et al., Phys. Rev. Lett. 86, 5866 (2001).

[6] V. S. Uma et al., Pram. J. Phys. 86, No. 1, 185 (2016).

[7] M. A. J. Mariscotti et al., Phys. Rev. 178 (4), 1864 (1969).

[8] A. Goel and A. K. Jain, Mod. Phys. Lett. A 29, 2403 (1990).

[9] H. Toki and A. Faessler, Nucl. Phys. A 253, 231 (1975).

[10] The ENSDF and XUNDL database available on BNL website at [http://www.nndc.bnl.gov]. 